\title{
An All-Photonic Molecular Amplifier and Binary Flip-flop
}

\author{
Thomas S. C. MacDonald, ${ }^{* \dagger \dagger}$ Timothy W. Schmidt, ${ }^{*, \dagger}$ and Jonathon E. \\ Beves $*, \dagger^{*}$ \\ $\dagger$ School of Chemistry, UNSW Sydney, NSW 2052, Australia \\ $\ddagger A R C$ Centre of Excellence in Exciton Science, School of Chemistry, UNSW Sydney, NSW 2052, \\ Australia \\ E-mail: t.macdonald@unsw.edu.au; timothy.schmidt@unsw.edu.au; \\ j.beves@unsw.edu.au
}




\begin{abstract}
A chemical system is proposed that is capable of amplifying small optical inputs into large changes in internal composition, based on a feedback interaction between switchable fluorescence and visible-light photoswitching. This system would demonstrate bifurcating reaction kinetics under irradiation and reach one of two stable photostationary states depending on the initial composition of the system. This behavior would allow the system to act as a chemical realization of the flip-flop circuit, the fundamental element in sequential logic and binary memory storage. We use detailed numerical modeling to demonstrate the feasibility of the proposed behavior based on known molecular phenomena, and comment on some of the conditions required to realize this system.
\end{abstract}

\title{
Graphical TOC Entry
}

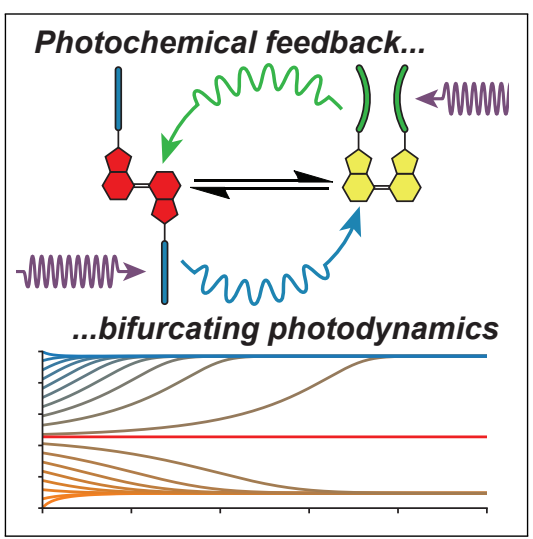




\section{Introduction}

Logic systems constructed from nano-scale chemical components have been a topic of discussion ${ }^{1}$ for much of the past century for reasons including component size, low power consumption, physical flexibility, solution processability, and the ability to respond to chemical species. At their most sophisticated such logic systems might resemble chemical computers, while less complex devices could be used for self-regulatory control of industrial processes by analogy to the regulatory reaction networks that govern enzyme activities in living organisms. ${ }^{2}$

Previous reports of molecular logic used chemical stimuli or light as inputs and/or outputs for molecular devices ${ }^{3-8}$ such as Boolean logic gates, ${ }^{9-14}$ multiplexers and demultiplexers, ${ }^{15}$ or memory components capable of storing and retrieving information. ${ }^{16,17}$ Despite successes in creating individual molecular devices, little progress has been made toward cascading multiple devices into more complex systems, and molecular computing remains a distant prospect. Two key requirements can be identified for molecular devices that can be assembled into arbitrarily larger systems: input-output homogeneity ${ }^{5,18}$ and constant signal amplitude. ${ }^{19}$ Input-output homogeneity refers to the use of a single information basis (e.g. photons or electrons) for both logical inputs and outputs.

Constant signal amplitude means that the intensity of a given signal must not diminish over multiple operations. As information processing necessarily comes with an energy cost, ${ }^{20}$ this implies that functional molecular logic devices will be dissipative systems ${ }^{21}$ that can only operate while 'fueled' by some external source of energy.

The problem of information amplitude would be solved by autoamplifying small perturbations away from an initially stable state. This process of amplification does work, and therefore requires an external source of energy (chemical, light, electrical, etc.). For example, solid-state electronic amplifiers use electrical energy to amplify a small signal voltage to produce a much larger output, while autoamplification of chirality ${ }^{22-29}$ requires the use of a chemical fuel to produce an enantioenriched product. Of these two examples the electronic amplifier has the advantage of using a general (electricity) rather than specialist chemical fuel source and produces no waste beyond heat. This lack of waste production allows the electronic voltage amplifier to operate continuously, 
while continuous operation of the chemical chirality amplifier requires the additional process of removing chemical waste products.

In one remarkable example of chiral autoamplification, a pyrimidyl alkanol with an initial enantiomeric excess (e.e.) as low as $5 \times 10^{-5} \%$ was used to establish an e.e. of $>99.5 \%$ after three cycles of chiral autoamplification. ${ }^{29}$ Chiral autocatalysis of this form is a kinetic rather than a thermodynamic phenomenon: ${ }^{30}$ in accordance with microscopic reversibility, a catalyst that asymmetrically catalyzes the formation of a chiral $R$ or $S$ product from a racemic starting material must also asymmetrically catalyze the return of the same chirality back to the racemic starting material. Chiral autoamplification thus requires both autocatalysis and a non-closed system in which the kinetic product can be removed or the reaction can be halted before the racemic equilibrium is eventually reached.

Compared to these autoamplifying chemical systems, an autoamplifying system based on photochemistry would have a number of advantages. First, any reaction network involving irreversible photochemical steps is not 'closed' thermodynamically and is not bound by microscopic reversibility. ${ }^{31}$ Light can be used by molecular devices to do work without producing any chemical waste, and emitted light can be used to transmit energy or information between spatially separated molecular devices. Finally, light can be delivered to chemical systems with high spatio-temporal precision and is non-persistent after the light source is turned off. These attributes make light an ideal medium for fueling and networking molecular devices. ${ }^{3,7,15,32-37}$

We propose a photoswitchable system in which each of the two isomers is autocatalytic with respect to light at the expense of the other. This behavior leads to the formation of two photostationary states (PSS) under irradiation by a single light source, each of which is locally stable within a range of compositions and separated from the other by a bifurcation point - a dynamically unstable composition from which both PSSs are equally accessible.

Small initial perturbations of composition about this bifurcation point are amplified into larger excesses by prolonged irradiation, comparable to chiral autoamplification. ${ }^{29}$ Irradiation of a system placed 'exactly' at the bifurcation point will lead to an indeterminate final outcome (i.e. either 
photostationary state might be reached). This system thus represents a light-powered bidirectional amplifier of chemical state. Finally, and in contrast to autocatalytic chemical amplifiers, this photochemical system could be reset to the unstable bifurcation point using suitably chosen light and thus is in principle indefinitely reusable.

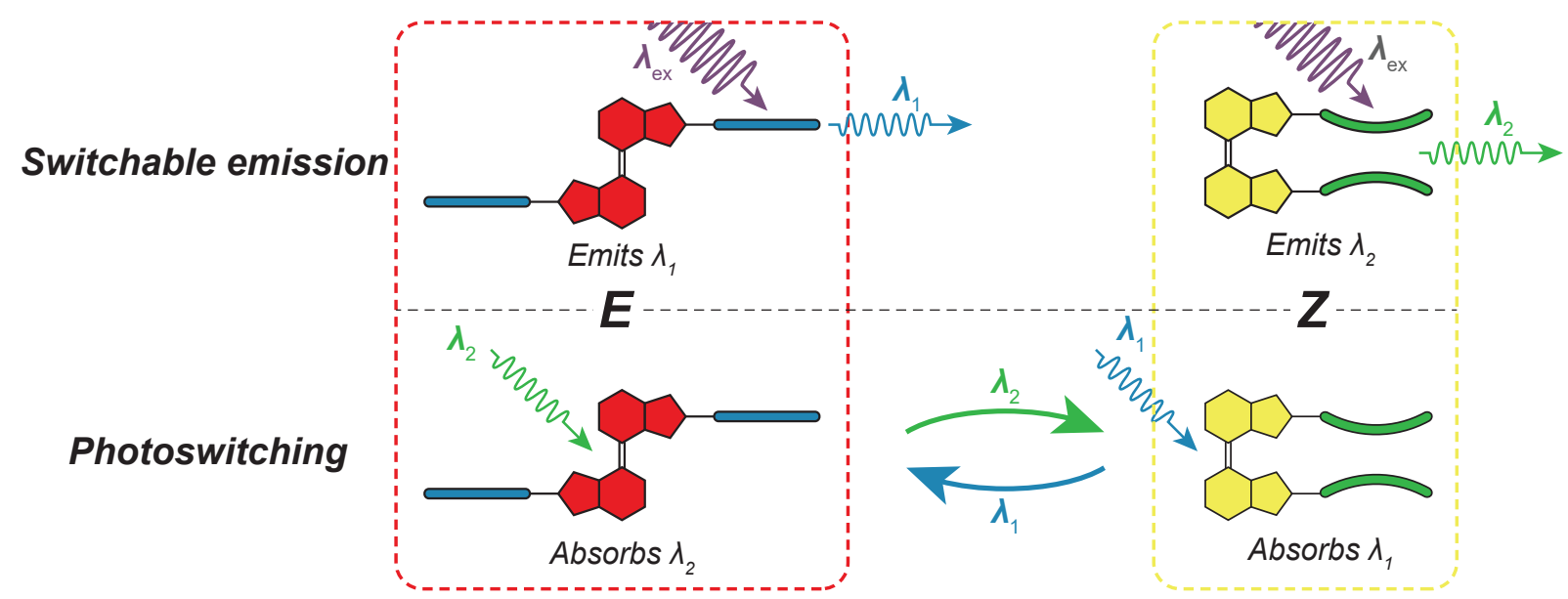

Figure 1. Autoamplification with a model photoswitch based on $E$ (left, red)/Z (right, yellow) isomerization. Top: each isomer is emissive under irradiation at $\lambda_{e x}$, but with different emission spectra due to excimer formation of the $Z$ isomer. Bottom: photoisomerization in each direction is driven by light emitted by the other isomer, making the system competitively autocatalytic under irradiation at $\lambda_{e x}$.

We propose a photoswitchable molecular system that also demonstrates photoswitchable light emission. ${ }^{38-48}$ External irradiation $\lambda_{e x}$ would be absorbed and re-emitted by each species with an emission spectrum that is preferentially re-absorbed by the other isomer. A schematic example of this feedback concept is shown in Figure 1 for two dye functionalities substituted onto an $E / Z$ photoswitch core ${ }^{49}$ in which $E \rightarrow Z$ photoisomerization leads to red-shifted excimer emission. ${ }^{50,51}$ The single emissive/responsive species discussed here is merely one approach towards all-photonic feedback: other approaches toward feedback might include switchable FRET or a photoacid paired with a $\mathrm{pH}$-responsive fluorescent dye, with the single requirement that the emission spectrum of each state must be selectively matched to the absorption spectra of the other state. 


\section{Modeling}

We assume a compound that can exist as an $E$ or a $Z$ photoisomer, similar to that shown in Figure 1. We then make the following key photophysical assumptions:

1. All isomerization is photoisomerization: there is no thermal pathway between $E$ and $Z$ on the experimental timescale.

2. The only intermolecular interaction is energy transfer via photon emission and (re)absorption. No aggregation or FRET-type energy transfer takes place (i.e. the system is infinitely dilute)

3. All quantum yields are independent of wavelength.

4. For each molecule, there is no intramolecular energy transfer between the emissive dyes and the photoswitchable core electronic subunits. Photoexcitation of the dyes or the photoswitch can respectively lead only to emission or isomerization, or nonradiative decay.

Assumptions 1-3 are physically realistic and could be achieved through the use of thermally bistable ('P-type') photoswitches and low concentrations. Assumption 4 is more problematic: while it is in principle possible to eliminate intramolecular energy transfer in violation of Kasha's rule through careful molecular design, ${ }^{52}$ we anticipate that doing so would be the major challenge in experimental realizations of this concept.

We consider a mixed system of two photoisomers, $E$ and $Z$ at total concentration $c_{0}$, and describe the state of this system by the mole fraction $x_{E}$ with $x_{E}+x_{Z}=1$. The spectral absorbance of the mixed system under external irradiation is given by the Beer-Lambert equation:

$$
A_{\text {ext }}(\lambda)=c_{0} l \sum_{i=E, Z} x_{i} \varepsilon_{i}(\lambda)
$$

where $\varepsilon_{i}(\lambda)$ is the spectral molar absorptivity of species $i$ over all wavelengths in $\mathrm{L} \mathrm{mol}^{-1} \mathrm{~cm}^{-1}$. The rate of change of composition of this system, $\dot{x}_{E}$, under irradiation with incident spectral photon flux $q_{\text {ext }}^{0}(\lambda)\left(\mathrm{mol} \mathrm{s}^{-1} \mathrm{~nm}^{-1}\right.$ of photons) will follow ${ }^{53}$ 


$$
\begin{aligned}
\dot{x}_{E} & =\int \frac{q_{e x t}^{0}\left(1-10^{-A_{e x t}}\right)}{c_{0} V}\left(\frac{x_{E} \varepsilon_{E} \phi_{E \rightarrow Z}^{i s o}-x_{Z} \varepsilon_{Z} \phi_{Z \rightarrow E}^{i s o}}{\sum_{i=E, Z} x_{i} \varepsilon_{i}^{\text {total }}}\right) \mathrm{d} \lambda \\
& =\int \frac{P_{e x t}}{c_{0}}\left(\frac{x_{E} \varepsilon_{E} \phi_{E \rightarrow Z}^{i s o}-x_{Z} \varepsilon_{Z} \phi_{Z \rightarrow E}^{i s o}}{\sum_{i=E, Z} x_{i} \varepsilon_{i}^{\text {total }}}\right) \mathrm{d} \lambda
\end{aligned}
$$

where $P_{\text {ext }}(\lambda)$ is the absorbed spectral photon flux density with units $\mathrm{mol} \mathrm{s}^{-1} \mathrm{~nm}^{-1} \mathrm{~L}^{-1},{ }^{54}$ i.e. the spectrum of photons being absorbed by the system, $V$ is the total sample volume, $A_{\text {ext }}(\lambda)$ the spectral absorbance as defined in Equation (1), $\varepsilon_{E}(\lambda)$ and $\varepsilon_{Z}(\lambda)$ are the spectral molar absorptivities of $E$ and $Z$ respectively with units $\mathrm{L} \mathrm{mol}^{-1} \mathrm{~cm}^{-1}$, and $\phi_{E \rightarrow Z}^{i s o}$ and $\phi_{Z \rightarrow E}^{i s o}$ the respective quantum yields for $E \rightarrow Z$ and $Z \rightarrow E$ photoisomerization.

We now turn to a system of the type shown in Figure 1. We assume that the electronic systems responsible for photoisomerization and for emission are entirely separate, allowing the total molar absorption spectrum $\varepsilon^{\text {total }}$ for each isomer $i$ to be separated into two molar absorbance spectra for the fluorescence and photoisomerizing parts of the molecule, $\varepsilon_{i}^{\text {total }}=\varepsilon_{i}^{F}+\varepsilon_{i}^{i s o}(i=E, Z)$.

First we model the 'internal' photon flux emitted upon irradiation. The spectral photon flux of emitted photons $q_{i n t}^{0}(\lambda)$ can be modeled as a weighted sum of the contributions from each isomer $i$ :

$$
q_{i n t}^{0}(\lambda)=\sum_{j=E, Z} \phi_{j}^{F} \overline{f_{j}}(\lambda) \int_{\lambda} q_{\text {ext }}^{0}\left(1-10^{-A_{\text {ext }}}\right) \cdot \frac{x_{j} \varepsilon_{j}^{F}}{\sum_{i=E, Z} x_{i} \varepsilon_{i}^{\text {total }}} \mathrm{d} \lambda
$$

with $A_{\text {ext }}(\lambda)$ the spectral absorbance as in Equation (1), and for each isomer $i \phi_{i}^{F}$ is the quantum yield for fluorescence and $\overline{f_{i}}(\lambda)$ the emission spectrum normalized to an integrated area of 1 over all wavelengths.

We can then combine Equation (2) with Equation (3) to express the composition-dependent change in composition of the $E-Z$ system under irradiation, internal emission, and re-absorption: 


$$
\begin{aligned}
\dot{x}_{E} & =-\int\left[\frac{q_{\text {ext }}^{0}\left[1-10^{-A_{e x t}}\right]+q_{i n t}^{0}\left[1-10^{-A_{\text {int }}}\right]}{c_{0} V}\right]\left(\frac{x_{E} \varepsilon_{E}^{i s o} \phi_{E \rightarrow Z}^{i s o}-x_{Z} \varepsilon_{Z}^{i s o} \phi_{Z \rightarrow E}^{i s o}}{\sum_{i=E, Z} x_{i} \varepsilon_{i}^{\text {total }}}\right) \mathrm{d} \lambda \\
& =-\int\left[\frac{P_{e x t}+P_{i n t}}{c_{0}}\right]\left(\frac{x_{E} \varepsilon_{E}^{i s o} \phi_{E \rightarrow Z}^{i s o}-x_{Z} \varepsilon_{Z}^{i s o} \phi_{Z \rightarrow E}^{i s o}}{\sum_{i=E, Z} x_{i} \varepsilon_{i}^{\text {total }}}\right) \mathrm{d} \lambda
\end{aligned}
$$

where

$$
P_{i=e x t, i n t}(\lambda)=\frac{q_{i}^{0}(\lambda)\left[1-10^{-A_{i}(\lambda)}\right]}{V}
$$

is the absorbed spectral photon flux density under incident spectral photon flux $q_{i}^{0}$ (either external or internal), with units $\mathrm{mol} \mathrm{s}^{-1} \mathrm{~nm}^{-1} \mathrm{~L}^{-1}$. Under this model photoisomerization can occur either as a result of the externally applied absorbed photon flux $\left(P_{\text {ext }}\right)$ or as a result of re-absorbed 'internal' photons $\left(P_{\text {int }}\right) . A_{\text {int }}(\lambda)=c_{0} z \sum_{i=E, Z} x_{i} \varepsilon_{i}$ is the effective absorbance of internally emitted photons, with $z$ the effective optical path length of the internally emitted photons. The internally emitted light may escape the system or may be re-absorbed with the escaping proportion of internally emitted light given by $10^{-A_{\text {int }}}$. The relationship between the optical path lengths for external irradiation $(l)$ and for internal emissions $(z)$ will reflect the geometry of the system under study, but for the sake of simplicity we set $z=l$ for our numerical model.

Equation (4) was used for a numerical model of the system shown in Figure 1. While our models are valid for arbitrary spectral parameters and could be applied to experimental data, for simplicity we chose to model absorption and emission spectra as Lorentzian curves of the form

$$
L(\lambda)=\frac{\alpha}{\pi}\left(\frac{\gamma / 2}{\left(\lambda-\lambda_{0}\right)^{2}+(\gamma / 2)^{2}}\right)
$$

where $\lambda_{0}$ is the central wavelength of the peak, $\gamma$ is the full width at half maximum, and $\alpha$ is the area under the peak. Parameters for these spectra were set as shown in Table 1. In addition, quantum yields for fluorescence $\phi^{F}$ and photoisomerization $\phi^{i s o}$ were respectively set to 0.7 and 0.5 (and equal for both $E$ and $Z$ isomers) and $c_{0}$ and $l$ were both set to 1 . It is important to note that these parameters are arbitrarily chosen to demonstrate the general behavior of this system 
and are not practical requirements. Spectral terms were defined numerically at integer-nanometer wavelengths from 300 to $700 \mathrm{~nm}$.

Table 1: Lorentzian parameters used for modeled absorption and emission spectra.

\begin{tabular}{lllll}
\hline Spectrum & $\lambda_{0} / \mathrm{nm}$ & $\gamma / \mathrm{nm}$ & $\alpha$ & $\varepsilon_{\max }(2 \alpha / \gamma \pi) / \mathrm{L} \mathrm{mol}^{-1} \mathrm{~cm}^{-1}$ \\
\hline$\varepsilon_{E}^{F}$ & 345 & 20 & $10^{6}$ & $3.2 \times 10^{4}$ \\
$\varepsilon_{Z}^{F}$ & 355 & 20 & $10^{6}$ & $3.2 \times 10^{4}$ \\
$\varepsilon_{E \rightarrow Z}^{i s o}$ & 510 & 30 & $7 \times 10^{5}$ & $1.5 \times 10^{4}$ \\
$\varepsilon_{Z}^{i s o} \rightarrow E$ & 405 & 20 & $8 \times 10^{5}$ & $2.6 \times 10^{4}$ \\
$f_{E}$ & 400 & 20 & $1^{a}$ & \\
\hline$f_{Z}$ & 500 & 40 & $1^{a}$ & \\
\hline
\end{tabular}

For composition-dependent models, $\dot{x}_{E}$ was calculated directly as a function of $x_{E}$ using Equation (4). For time-dependent studies, $x_{E}(t)$ was calculated numerically.

\section{Results}

The response of the system to irradiation is shown in Figure 2 as the rate of change of composition of the switch against composition. As for standard photoswitches, irradiation of a sample at any composition at wavelengths that are absorbed directly by the photoswitch (Figure 2a) drives the system towards a single wavelength-dependent photostationary state where $\dot{x}_{E}=0$ (Figure 2c).

Qualitatively different behavior is seen upon irradiation at wavelengths absorbed and re-emitted by the molecule (Figure 2b), which results in three compositions where $\dot{x}_{E}=0$ (Figure $2 \mathrm{~d}$ ). Two of these stationary points are stable photostationary states, which we will denote here as $x_{E}^{P S S 1,2}$. A system placed at one of these compositions and then perturbed slightly away will experience a 'returning force' under irradiation and its composition $x_{E}$ will return to that local $x_{E}^{P S S}$. One stationary point is an unstable bifurcation point, $x_{E}^{*}$ : a system placed exactly at this composition is stable under irradiation, but any small perturbation will be amplified under irradiation as the system diverges away from $x_{E}^{*}$ and towards the locally stable composition $\left(x_{E}^{P S S 1}\right.$ or $\left.x_{E}^{P S S 2}\right)$. The behavior of this system under extended irradiation will thus move towards one of two different-but locally 

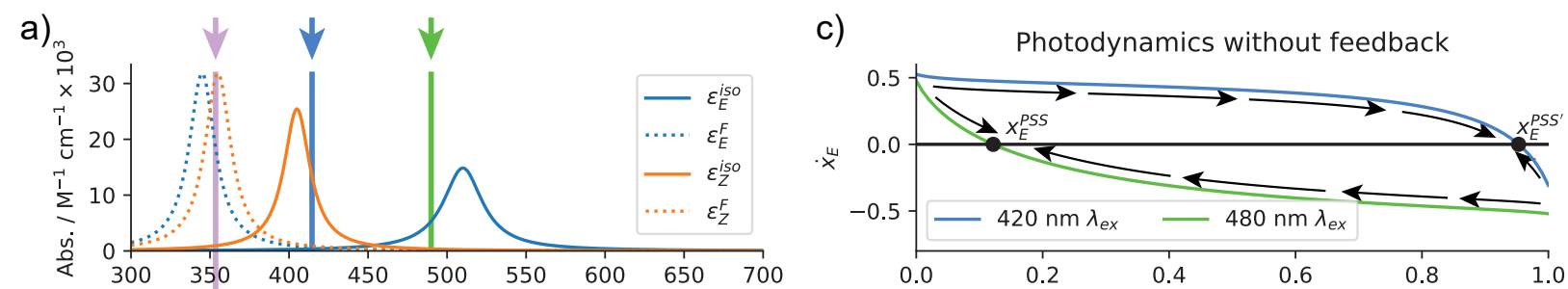

b)
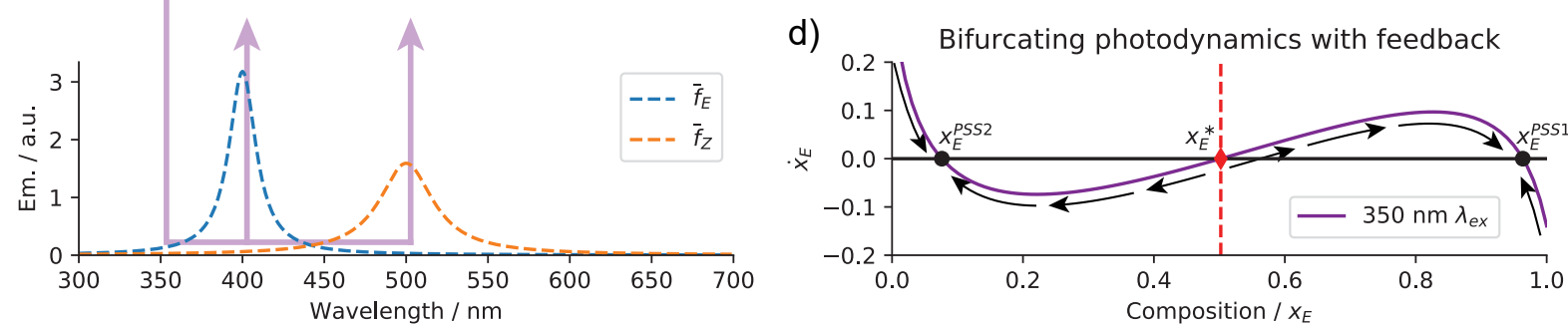

Figure 2. Simulated absorption (a) and emission (b) spectra defined using the parameters in Table 1. c) Direct irradiation of the photochromic system $\left(\lambda_{e x}=420\right.$ or $480 \mathrm{~nm}$, blue or green lines and arrows respectively) leads to a single wavelength-dependent photostationary state $x^{P S S}$ where $\dot{x}_{E}=0(\bullet)$ d) Irradiation at $350 \mathrm{~nm}$ (purple lines and arrows) drives indirect switching via re-emitted light, leading to three compositions where $\dot{x}_{E}=0$. Two are dynamically stable photostationary states $x_{E}^{P S S 1}$ and $x_{E}^{P S S 2}(\bullet)$ and one is a dynamically unstable bifurcation point $x_{E}^{*}$ $(\diamond)$.

stable-photostationary states with composition $x_{E}^{P S S 1}$ or $x_{E}^{P S S 2}$, depending on whether the initial state of the system was more or less $E$-rich than $x_{E}^{*}$.

These different behaviors of the system in response to irradiation at 454 or $350 \mathrm{~nm}$ are shown in Figure 3 for a variety of initial compositions. In Figure 3a irradiation at $454 \mathrm{~nm}$ drives all initial compositions towards a single PSS over time, while in Figure $3 \mathrm{~b}$ irradiation at $350 \mathrm{~nm}$ drives the system toward one of two distinct PSSs depending on initial composition. The $454 \mathrm{~nm}$ irradiation simulated here demonstrates another key functionality of this system: the PSS reached under 454 $\mathrm{nm}$ irradiation has the same composition as the bifurcation point under $350 \mathrm{~nm}$ irradiation. This makes it possible to 'reset' the system to a bifurcating state on demand, and we thus refer to this irradiation wavelength as $\lambda_{\text {reset }}$.

The presence of a bifurcation point $x_{E}^{*}$ makes this system capable of amplifying weak perturbations away from $x_{E}^{*}$ into larger binary signals. Crucially, this weak input is the same as the output of the system: a small input flux of blue or green $(<454$ or $>454 \mathrm{~nm})$ photons will, after amplification, result in a larger output of blue or green photons. This amplification requires that 
a)

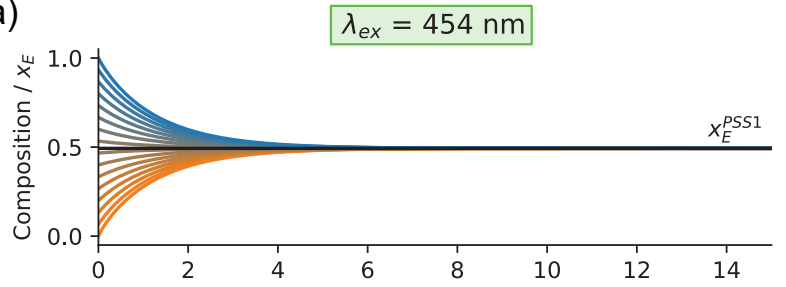

b)

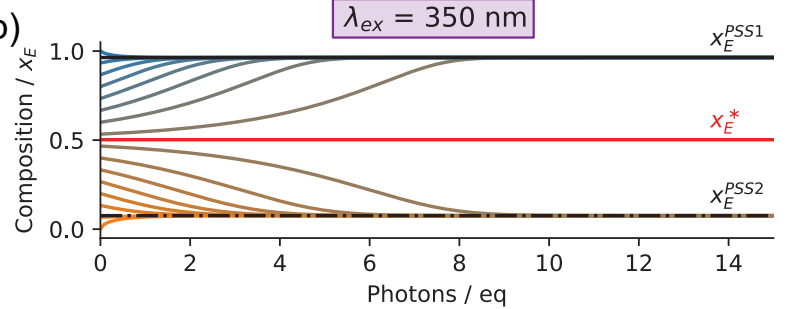

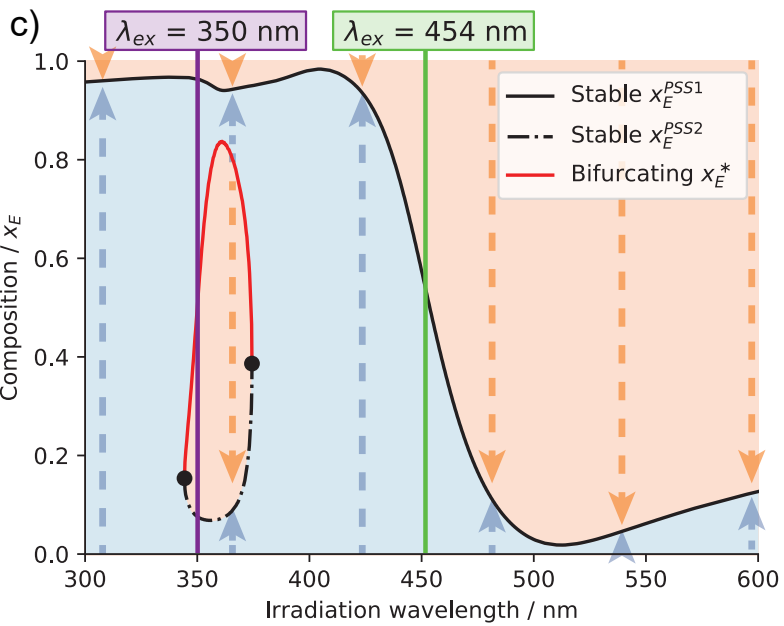

Figure 3. Changes in composition on irradiation. a) $454 \mathrm{~nm}$ irradiation does not cause internal fluorescence and the system approaches a single global PSS $x_{E}^{P S S 1}$. b) $350 \mathrm{~nm}$ irradiation drives the system towards one of two stable states, $x_{E}^{P S S 1}$ or $x_{E}^{P S S 2}$, depending on the initial composition. Stable $\left(x_{E}^{P S S}\right)$ and unstable $\left(x_{E}^{*}\right)$ stationary states are shown with black and red horizontal lines, respectively. c) Plotting stationary states as a function of irradiation wavelength $\lambda_{e x}$ reveals a landscape of compositions with $\dot{x_{E}}<0$ (shaded orange, downwards arrows), $\dot{x_{E}}>0$ (shaded blue, upwards arrows), and $\dot{x_{E}}=0$ (stationary points, see legend). Multiple stable states occur only for a limited range of irradiation wavelengths, here $345 \mathrm{~nm}<\lambda_{e x}<372 \mathrm{~nm}$. The $\lambda_{e x}$ used in (a) and (b) are indicated with vertical colored lines.

the system initially be placed in a composition as close to $x_{E}^{*}$ as possible, which can be achieved through direct visible-light photoisomerization of the system using a wavelength $\lambda_{\text {reset }}$ chosen such that the resultant PSS $x_{E}^{P S S}\left(\lambda_{\text {reset }}\right)$ is the same as the bifurcation point under $350 \mathrm{~nm}$ irradiation, as shown in Figure 3a.

It is interesting to note that the system achieves equilibrium far more quickly in Figure $3 \mathrm{a}$ (without feedback) than in Figure $3 b$ (with feedback). This is partially due to the decreased overall quantum efficiency resulting from imperfect emission $\left(\phi^{F}<1\right)$, but a more important factor is that the system is always close to dynamic equilibrium between compositions $x_{E}^{P S S 1}$ and $x_{E}^{P S S 2}$ : at compositions close to $x_{E}^{*}$, the rates of forward and backward photoisomerization almost balance one another and overall movement towards the local PSS is slow and photon-inefficient (see also the low magnitude of $\left|\dot{x}_{e}\right|$ in Figure $2 \mathrm{~d}$ vs Figure 2c). This is equivalent to the slow initial increase of enantiomeric excess seen in chiral autocatalysis of near-racemic mixtures. ${ }^{29}$

Each time amplification occurs, a single bit of information ('blue' or 'green') is stored and 
remains stored until the system is reset by irradiation at $\lambda_{\text {reset }}$. This memory is stable in the absence of energy input and decays only according to the thermal lifetime of the photoswitch in use, potentially leading to lifetimes of thousands of years. ${ }^{55}$ At any point, the memory can be re-amplified and read by irradiation with $350 \mathrm{~nm}$ light $\left(\lambda_{e x}\right)$.

The behavior is analogous to a flip-flop electronic circuit, also known as a bistable multivibrator or latch. ${ }^{20,56}$ Flip-flops are the fundamental storage element in sequential logic, allowing a bit of information to be stored and later accessed as required. While this is not the first example of an allphotonic molecular flip-flop, ${ }^{14,57-60}$ it is the first proposal to use the same wavelengths of light for inputs and outputs ('input-output homogeneity' ${ }^{5}$ ) and to incorporate light-powered amplification, allowing weak signals to be cascaded and 'wired' to further operations without loss of intensity.

Operation of the feedback system as a flip-flop is shown schematically in Figure 4. Beginning with the system at the bifurcation point, $x_{E}^{*}$ a binary input signal ('blue' or 'green') can be amplified and read out under irradiation at $\lambda_{e x}(350 \mathrm{~nm})$. The bit of information stored can be retained in the dark for an extended period and read out later as needed. When needed, the bit can be rewritten by a) irradiating at $\lambda_{\text {reset }}(454 \mathrm{~nm})$ to reset the system to $x_{E}^{*}$, followed by b) perturbation with an input and amplification under irradiation by $\lambda_{e x}$.

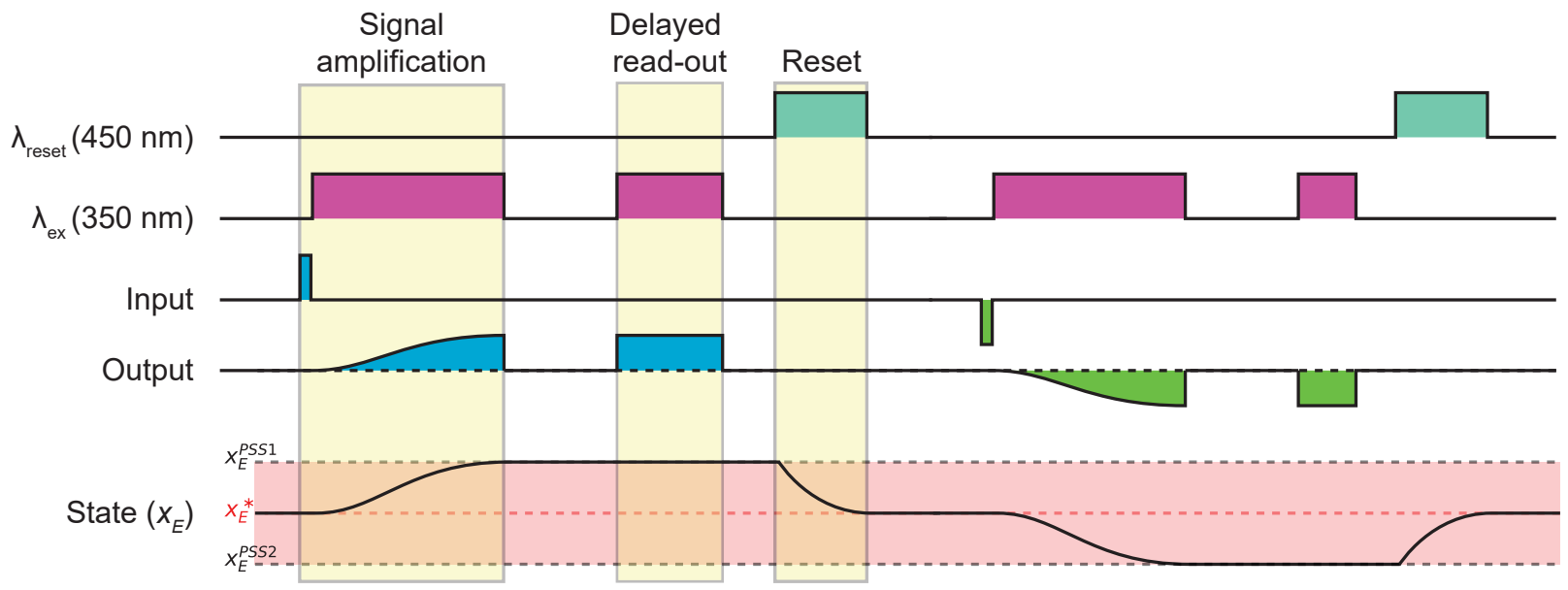

Figure 4. Input amplification and non-volatile memory storage. Starting with a system at the bifurcation point $x_{E}^{*}$, a small input (e.g. blue light) is amplified by irradiation at $\lambda_{e x}$ and leads to a larger output. The internal state is non-volatile and may be 'read out' later in time. Irradiation at $\lambda_{\text {reset }}$ resets the system to its bifurcation point $x_{E}^{*}$, allowing subsequent reuse. 


\section{Conclusions}

We present a theoretical study of an all-photonic feedback system that is effectively autocatalytic with respect to light. This requires combining the known phenomena of visible-light photoswitching $^{61-67}$ and switchable emission ${ }^{40,43,46,68,69}$ to produce a photoswitchable system involving two states. Each state is fluorescent under UV irradiation with an emission spectrum selectively absorbed by the other state, leading to competitive autoamplification of each photoisomer at the expense of the other. Using numerical modeling, we demonstrate that such an auto-amplifying system would be capable of bifurcation and bistability under irradiation, and could be used to amplify initially small differences in composition. This behavior is analogous to an electronic flipflop circuit, the fundamental component of sequential logic and one of the two logic components required to build a computer and process arbitrary information.

This is the first proposal of a molecular flip-flop incorporating amplification (the output signal is always the same, regardless of the amplitude of the input) and with homogeneous inputs and outputs (binary blue/green inputs result in binary blue/green outputs).

\section{Methods}

Models were written in Python 3.7 using the NumPy ${ }^{70}$ package and plotted using the MatPlotLib ${ }^{71}$ package.

\section{Acknowledgments}

We thank Prof. R. Dean Astumian and Prof. Joakim Andréasson for fruitful discussions. This work was supported by the Australian Research Council (Centre of Excellence in Exciton Science CE170100026 to T.W.S., FT170100094 to J.E.B.) and by the Australian Government (T.S.C.M., Australian Postgraduate Award). 


\section{References}

(1) Feynman, R. P. There's Plenty of Room at the Bottom (Transcript of Talk Given on December 29, 1959, at the Annual Meeting of the American Physical Society). Caltech Eng. Sci. 1960, $23,22-36$.

(2) Erbas-Cakmak, S.; Kolemen, S.; Sedgwick, A. C.; Gunnlaugsson, T.; James, T. D.; Yoon, J.; Akkaya, E. U. Molecular Logic Gates: The Past, Present and Future. Chem. Soc. Rev. 2018, 47, 2228-2248.

(3) Andréasson, J.; Pischel, U.; Straight, S. D.; Moore, T. A.; Moore, A. L.; Gust, D. All-Photonic Multifunctional Molecular Logic Device. J. Am. Chem. Soc. 2011, 133, 11641-11648.

(4) Pischel, U.; Andréasson, J.; Gust, D.; Pais, V. F. Information Processing with Molecules-Quo Vadis? ChemPhysChem 2013, 14, 28-46.

(5) Andréasson, J.; Pischel, U. Molecules with a Sense of Logic: A Progress Report. Chem. Soc. Rev. 2015, 44, 1053-1069.

(6) Pischel, U. Chemical Approaches to Molecular Logic Elements for Addition and Subtraction. Angew. Chem. Int. Ed. 2007, 46, 4026-4040.

(7) Silvi, S.; Constable, E. C.; Housecroft, C. E.; Beves, J. E.; Dunphy, E. L.; Tomasulo, M.; Raymo, F. M.; Credi, A. All-Optical Integrated Logic Operations Based on Chemical Communication between Molecular Switches. Chem. Eur. J. 2009, 15, 178-185.

(8) Silvi, S.; Venturi, M.; Credi, A. Light Operated Molecular Machines. Chem. Commun. 2011, 47, 2483-2489.

(9) de Silva, P. A.; Gunaratne, N. H. Q.; McCoy, C. P. A Molecular Photoionic AND Gate Based on Fluorescent Signalling. Nature 1993, 364, 42-44.

(10) Balzani, V.; Venturi, M.; Credi, A. Molecular Devices and Machines: A Journey into the Nano World; Wiley-VCH: Weinheim, 2003. 
(11) de Silva, A. P.; McClenaghan, N. D. Molecular-Scale Logic Gates. Chem. Eur. J. 2004, 10, $574-586$.

(12) Andréasson, J.; Terazono, Y.; Albinsson, B.; Moore, T. A.; Moore, A. L.; Gust, D. Molecular AND Logic Gate Based on Electric Dichroism of a Photochromic Dihydroindolizine. Angew. Chem. Int. Ed. 2005, 44, 7591-7594.

(13) Szaciłowski, K.; Macyk, W.; Stochel, G. Light-Driven OR and XOR Programmable Chemical Logic Gates. J. Am. Chem. Soc. 2006, 128, 4550-4551.

(14) Remón, P.; Bälter, M.; Li, S.; Andréasson, J.; Pischel, U. An All-Photonic Molecule-Based D Flip-Flop. J. Am. Chem. Soc. 2011, 133, 20742-20745.

(15) Amelia, M.; Baroncini, M.; Credi, A. A Simple Unimolecular Multiplexer/Demultiplexer. Angew. Chem. Int. Ed. 2008, 47, 6240-6243.

(16) Silvi, S.; Arduini, A.; Pochini, A.; Secchi, A.; Tomasulo, M.; Raymo, F. M.; Baroncini, M.; Credi, A. A Simple Molecular Machine Operated by Photoinduced Proton Transfer. J. Am. Chem. Soc. 2007, 129, 13378-13379.

(17) Avellini, T.; Li, H.; Coskun, A.; Barin, G.; Trabolsi, A.; Basuray, A. N.; Dey, S. K.; Credi, A.; Silvi, S.; Stoddart, J. F. et al. Photoinduced Memory Effect in a Redox Controllable Bistable Mechanical Molecular Switch. Angew. Chem. Int. Ed. 2012, 51, 1611-1615.

(18) Ling, J.; Daly, B.; D. Silverson, V. A.; de Silva, A. P. Taking Baby Steps in Molecular LogicBased Computation. Chem. Commun. 2015, 51, 8403-8409.

(19) Baroncini, M.; Semeraro, M.; Credi, A. Unconventional Nonlinear Input-Output Response in a Luminescent Molecular Switch by Inner Filtering Effects. ChemPhysChem 2017, 18, 1755-1759.

(20) Shannon, C. E. A Mathematical Theory of Communication. Bell Syst. Tech. J. 1948, 27, 379423. 
(21) Nicolis, G.; Prigogine, I. Self-Organization in Nonequilibrium Systems: From Dissipative Structures to Order through Fluctuations; Wiley: New York, 1977.

(22) Soai, K.; Niwa, S.; Hori, H. Asymmetric Self-Catalytic Reaction. Self-Production of Chiral 1(3-Pyridyl)Alkanols as Chiral Self-Catalysts in the Enantioselective Addition of Dialkylzinc Reagents to Pyridine-3-Carbaldehyde. J. Chem. Soc., Chem. Commun. 1990, 982-983.

(23) Soai, K.; Shibata, T.; Morioka, H.; Choji, K. Asymmetric Autocatalysis and Amplification of Enantiomeric Excess of a Chiral Molecule. Nature 1995, 378, 767-768.

(24) Soai, K.; Shibata, T.; Sato, I. Enantioselective Automultiplication of Chiral Molecules by Asymmetric Autocatalysis. Acc. Chem. Res. 2000, 33, 382-390.

(25) Kondepudi, D. K.; Asakura, K. Chiral Autocatalysis, Spontaneous Symmetry Breaking, and Stochastic Behavior. Acc. Chem. Res. 2001, 34, 946-954.

(26) Singleton, D. A.; Vo, L. K. Enantioselective Synthesis without Discrete Optically Active Additives. J. Am. Chem. Soc. 2002, 124, 10010-10011.

(27) Gridnev, I. D.; Serafimov, J. M.; Quiney, H.; Brown, J. M. Reflections on Spontaneous Asymmetric Synthesis by Amplifying Autocatalysis. Org. Biomol. Chem. 2003, 1, 3811-3819.

(28) Soai, K.; Sato, I.; Shibata, T.; Komiya, S.; Hayashi, M.; Matsueda, Y.; Imamura, H.; Hayase, T.; Morioka, H.; Tabira, H. et al. Asymmetric Synthesis of Pyrimidyl Alkanol without Adding Chiral Substances by the Addition of Diisopropylzinc to Pyrimidine-5Carbaldehyde in Conjunction with Asymmetric Autocatalysis. Tetrahedron Asymmetry 2003, 14, 185-188.

(29) Sato, I.; Urabe, H.; Ishiguro, S.; Shibata, T.; Soai, K. Amplification of Chirality from Extremely Low to Greater than $99.5 \%$ Ee by Asymmetric Autocatalysis. Angew. Chem. Int. Ed. 2003, 42, 315-317. 
(30) Blackmond, D. G. "If Pigs Could Fly" Chemistry: A Tutorial on the Principle of Microscopic Reversibility. Angew. Chem. Int. Ed. 2009, 48, 2648-2654.

(31) Astumian, R. D. Microscopic Reversibility as the Organizing Principle of Molecular Machines. Nat. Nanotechnol. 2012, 7, 684-688.

(32) Andréasson, J.; Kodis, G.; Terazono, Y.; Liddell, P. A.; Bandyopadhyay, S.; Mitchell, R. H.; Moore, T. A.; Moore, A. L.; Gust, D. Molecule-Based Photonically Switched Half-Adder. J. Am. Chem. Soc. 2004, 126, 15926-15927.

(33) Credi, A. Molecules That Make Decisions. Angew. Chem. Int. Ed. 2007, 46, 5472-5475.

(34) Balzani, V.; Credi, A.; Venturi, M. Photochemical Conversion of Solar Energy. ChemSusChem 2008, 1, 26-58.

(35) Kärnbratt, J.; Hammarson, M.; Li, S.; Anderson, H. L.; Albinsson, B.; Andréasson, J. Photochromic Supramolecular Memory With Nondestructive Readout. Angew. Chem. Int. Ed. 2010, 49, 1854-1857.

(36) Bälter, M.; Li, S.; Nilsson, J. R.; Andréasson, J.; Pischel, U. An All-Photonic Molecule-Based Parity Generator/Checker for Error Detection in Data Transmission. J. Am. Chem. Soc. 2013, $135,10230-10233$.

(37) Ceroni, P.; Credi, A.; Venturi, M. Light to Investigate (Read) and Operate (Write) Molecular Devices and Machines. Chem. Soc. Rev. 2014, 43, 4068-4083.

(38) Wang, J.; Kulago, A.; Browne, W. R.; Feringa, B. L. Photoswitchable Intramolecular HStacking of Perylenebisimide. J. Am. Chem. Soc. 2010, 132, 4191-4196.

(39) Díaz, S. A.; Giordano, L.; Jovin, T. M.; Jares-Erijman, E. A. Modulation of a Photoswitchable Dual-Color Quantum Dot Containing a Photochromic FRET Acceptor and an Internal Standard. Nano Lett. 2012, 12, 3537-3544. 
(40) Kim, S.; Yoon, S.-J.; Park, S. Y. Highly Fluorescent Chameleon Nanoparticles and Polymer Films: Multicomponent Organic Systems That Combine FRET and Photochromic Switching. J. Am. Chem. Soc. 2012, 134, 12091-12097.

(41) Zhu, L.; Li, X.; Zhang, Q.; Ma, X.; Li, M.; Zhang, H.; Luo, Z.; Ågren, H.; Zhao, Y. Unimolecular Photoconversion of Multicolor Luminescence on Hierarchical Self-Assemblies. J. Am. Chem. Soc. 2013, 135, 5175-5182.

(42) Bu, J.; Watanabe, K.; Hayasaka, H.; Akagi, K. Photochemically Colour-Tuneable White Fluorescence Illuminants Consisting of Conjugated Polymer Nanospheres. Nat. Commun. 2014, 5,3799 .

(43) Bälter, M.; Li, S.; Morimoto, M.; Tang, S.; Hernando, J.; Guirado, G.; Irie, M.; Raymo, F. M.; Andréasson, J. Emission Color Tuning and White-Light Generation Based on Photochromic Control of Energy Transfer Reactions in Polymer Micelles. Chem. Sci. 2016, 7, 5867-5871.

(44) Irie, M.; Morimoto, M. Photoswitchable Turn-on Mode Fluorescent Diarylethenes: Strategies for Controlling the Switching Response. BCSJ 2017, 91, 237-250.

(45) Kim, D.; Park, S. Y. Multicolor Fluorescence Photoswitching: Color-Correlated versus Color-Specific Switching. Adv. Opt. Mater. 2018, 6, 1800678.

(46) Naren, G.; Hsu, C.-W.; Li, S.; Morimoto, M.; Tang, S.; Hernando, J.; Guirado, G.; Irie, M.; Raymo, F. M.; Sundén, H. et al. An All-Photonic Full Color RGB System Based on Molecular Photoswitches. Nat. Commun. 2019, 10, 1-7.

(47) Johnstone, M. D.; Hsu, C.-W.; Hochbaum, N.; Andréasson, J.; Sundén, H. Multi-Color Emission with Orthogonal Input Triggers from a Diarylethene Pyrene-OTHO Organogelator Cocktail. Chem. Commun. 2020, 56, 988-991.

(48) Naren, G.; Li, S.; Andréasson, J. A Simplicity-Guided Cocktail Approach toward Multicolor Fluorescent Systems. Chem. Commun. 2020, 56, 3377-3380. 
(49) Russew, M.-M.; Hecht, S. Photoswitches: From Molecules to Materials. Adv. Mater. 2010, $22,3348-3360$.

(50) Lister, F. G. A.; Bailly, B. A. F. L.; Webb, S. J.; Clayden, J. Ligand-Modulated Conformational Switching in a Fully Synthetic Membrane-Bound Receptor. Nat. Chem. 2017, 9, $420-425$.

(51) Lister, F. G. A.; Eccles, N.; Pike, S. J.; Brown, R. A.; Whitehead, G. F. S.; Raftery, J.; Webb, S. J.; Clayden, J. Bis-Pyrene Probes of Foldamer Conformation in Solution and in Phospholipid Bilayers. Chem. Sci. 2018, 9, 6860-6870.

(52) Qian, H.; Cousins, M. E.; Horak, E. H.; Wakefield, A.; Liptak, M. D.; Aprahamian, I. Suppression of Kasha's Rule as a Mechanism for Fluorescent Molecular Rotors and AggregationInduced Emission. Nat. Chem. 2017, 9, 83-87.

(53) Bolton, J. R. A Master Equation for Photochemical Rates. Photochem. Photobiol. 2020, php. 13325.

(54) Nič, M., Jirát, J., Košata, B., Jenkins, A., McNaught, A., Eds. IUPAC Compendium of Chemical Terminology: Gold Book, 2nd ed.; IUPAC: Research Triagle Park, NC, 2009.

(55) Ryabchun, A.; Li, Q.; Lancia, F.; Aprahamian, I.; Katsonis, N. Shape-Persistent Actuators from Hydrazone Photoswitches. J. Am. Chem. Soc. 2019, 141, 1196-1200.

(56) Clayton, G. B.; Winder, S. Operational Amplifiers, 5th ed.; Newnes: Oxford ; Boston, 2003.

(57) Baron, R.; Onopriyenko, A.; Katz, E.; Lioubashevski, O.; Willner, I.; Wang, S.; Tian, H. An Electrochemical/Photochemical Information Processing System Using a MonolayerFunctionalized Electrode. Chem. Commun. 2006, 2147-2149.

(58) de Ruiter, G.; Motiei, L.; Choudhury, J.; Oded, N.; van der Boom, M. E. Electrically Addressable Multistate Volatile Memory with Flip-Flop and Flip-Flap-Flop Logic Circuits on a Solid Support. Angew. Chem. Int. Ed. 2010, 49, 4780-4783. 
(59) Pischel, U.; Andréasson, J. A Simplicity-Guided Approach toward Molecular Set-Reset Memories. New J. Chem. 2010, 34, 2701-2703.

(60) de Ruiter, G.; van der Boom, M. E. Sequential Logic and Random Access Memory (RAM): A Molecular Approach. J. Mater. Chem. 2011, 21, 17575-17581.

(61) Siewertsen, R.; Neumann, H.; Buchheim-Stehn, B.; Herges, R.; Näther, C.; Renth, F.; Temps, F. Highly Efficient Reversible Z-E Photoisomerization of a Bridged Azobenzene with Visible Light through Resolved S1(N $\left.\pi^{*}\right)$ Absorption Bands. J. Am. Chem. Soc. 2009, 131, $15594-15595$.

(62) Bléger, D.; Schwarz, J.; Brouwer, A. M.; Hecht, S. O -Fluoroazobenzenes as Readily Synthesized Photoswitches Offering Nearly Quantitative Two-Way Isomerization with Visible Light. J. Am. Chem. Soc. 2012, 134, 20597-20600.

(63) Samanta, S.; Beharry, A. A.; Sadovski, O.; McCormick, T. M.; Babalhavaeji, A.; Tropepe, V.; Woolley, G. A. Photoswitching Azo Compounds in Vivo with Red Light. J. Am. Chem. Soc. 2013, 135, 9777-9784.

(64) Helmy, S.; Oh, S.; Leibfarth, F. A.; Hawker, C. J.; Read de Alaniz, J. Design and Synthesis of Donor-Acceptor Stenhouse Adducts: A Visible Light Photoswitch Derived from Furfural. J. Org. Chem. 2014, 79, 11316-11329.

(65) Helmy, S.; Leibfarth, F. A.; Oh, S.; Poelma, J. E.; Hawker, C. J.; Read de Alaniz, J. Photoswitching Using Visible Light: A New Class of Organic Photochromic Molecules. J. Am. Chem. Soc. 2014, 136, 8169-8172.

(66) Knie, C.; Utecht, M.; Zhao, F.; Kulla, H.; Kovalenko, S.; Brouwer, A. M.; Saalfrank, P.; Hecht, S.; Bléger, D. Ortho-Fluoroazobenzenes: Visible Light Switches with Very LongLived Z Isomers. Chem. Eur. J. 2014, 20, 16492-16501. 
(67) Dragu, E. A.; Ion, A. E.; Shova, S.; Bala, D.; Mihailciuc, C.; Voicescu, M.; Ionescu, S.; Nica, S. Visible-Light Triggered Photoswitching Systems Based on Fluorescent AzulenylSubstituted Dithienylcyclopentenes. RSC Adv. 2015, 5, 63282-63286.

(68) Li, C.; Zhang, Y.; Hu, J.; Cheng, J.; Liu, S. Reversible Three-State Switching of Multicolor Fluorescence Emission by Multiple Stimuli Modulated FRET Processes within Thermoresponsive Polymeric Micelles. Angew. Chem. Int. Ed. 2010, 49, 5120-5124.

(69) Karpiuk, J.; Karolak, E.; Nowacki, J. Tuneable White Fluorescence from Intramolecular Exciplexes. Phys. Chem. Chem. Phys. 2010, 12, 8804-8809.

(70) Harris, C. R.; Millman, K. J.; van der Walt, S. J.; Gommers, R.; Virtanen, P.; Cournapeau, D.; Wieser, E.; Taylor, J.; Berg, S.; Smith, N. J. et al. Array Programming with NumPy. Nature 2020, 585, 357-362.

(71) Hunter, J. D. Matplotlib: A 2D Graphics Environment. Comput. Sci. Eng. 2007, 9, 90-95. 